ANNALES

POLONICI MATHEMATICI

$97.2(2010)$

\title{
Zeros of solutions of certain higher order linear differential equations
}

by Hong-Yan Xu (Jingdezhen) and CAI-Feng Yi (Nanchang)

\begin{abstract}
We investigate the exponent of convergence of the zero-sequence of solutions of the differential equation

$$
f^{(k)}+a_{k-1}(z) f^{(k-1)}+\cdots+a_{1}(z) f^{\prime}+D(z) f=0
$$

where $D(z)=Q_{1}(z) e^{P_{1}(z)}+Q_{2}(z) e^{P_{2}(z)}+Q_{3}(z) e^{P_{3}(z)}, P_{1}(z), P_{2}(z), P_{3}(z)$ are polynomials of degree $n \geq 1, Q_{1}(z), Q_{2}(z), Q_{3}(z), a_{j}(z)(j=1, \ldots, k-1)$ are entire functions of order less than $n$, and $k \geq 2$.
\end{abstract}

1. Introduction and results. We shall assume that the reader is familiar with the fundamental results and the standard notations of the Nevanlinna value distribution theory of meromorphic functions (see [5, 8]). We will use the notation $\sigma(f)$ to denote the order of growth of a meromorphic function $f(z)$ and $\lambda(f)$ to denote the exponent of convergence of the zero-sequence of $f(z)$.

K. Ishizaki and K. Tohge [6, 7] have studied the exponent of convergence of the zero-sequence of solutions of the equation

$$
f^{\prime \prime}+\left(e^{P_{1}(z)}+e^{P_{2}(z)}+Q_{0}(z)\right) f=0,
$$

where $P_{1}(z), P_{2}(z)$ are non-constant polynomials

$$
P_{1}(z)=\zeta_{1} z^{n}+\cdots, \quad P_{2}(z)=\zeta_{2} z^{m}+\cdots, \quad \zeta_{1} \zeta_{2} \neq 0 \quad(n, m \in \mathbb{N}) .
$$

and $Q_{0}(z)$ is an entire function of order less than $\max \{n, m\}$, and $e^{P_{1}(z)}$ and $e^{P_{2}(z)}$ are linearly independent. They have obtained the following results:

Theorem A ([7]). Suppose that $n=m$, and that $\zeta_{1} \neq \zeta_{2}$ in (2). If $\zeta_{1} / \zeta_{2}$ is non-real, then for any solution $f \not \equiv 0$ of $(2)$, we have $\lambda(f)=\infty$.

Theorem B ([6]). Suppose that $n=m$, and that $\zeta_{1} / \zeta_{2}=\rho>0$ in (2). If $0<\rho<1 / 2$ or $Q_{0}(z) \equiv 0,3 / 4<\rho<1$, then for any solution $f \not \equiv 0$ of $(2)$, we have $\lambda(f) \geq n$.

2010 Mathematics Subject Classification: 34M10, 30D35.

Key words and phrases: linear differential equation, entire function, zero. 
Recently, J. Tu and Z. X. Chen [9] investigated the exponent of convergence of the zero-sequence of solutions of the differential equation

$$
f^{\prime \prime}+\left(Q_{1}(z) e^{P_{1}(z)}+Q_{2}(z) e^{P_{2}(z)}+Q_{3}(z) e^{P_{3}(z)}\right) f=0,
$$

and obtained the following theorem:

Theorem C ([9]). Let $Q_{1}, Q_{2}, Q_{3}$ be entire functions of order less than $n$, and $P_{1}(z), P_{2}(z), P_{3}(z)$ be polynomials of degree $n \geq 1$,

$$
P_{1}(z)=\zeta_{1} z^{n}+\cdots, \quad P_{2}(z)=\zeta_{2} z^{n}+\cdots, \quad P_{3}(z)=\zeta_{3} z^{n}+\cdots,
$$

where $\zeta_{1}, \zeta_{2}, \zeta_{3}$ are complex numbers.

(i) If $\zeta_{1} / \zeta_{2}$ is non-real and $0<\lambda=\zeta_{3} / \zeta_{2}<1 / 2$, then for any solution $f \not \equiv 0$ of $(3)$, we have $\lambda(f)=\infty$.

(ii) If $0<\zeta_{1} / \zeta_{2}<1 / 4$ and $0<\lambda=\zeta_{3} / \zeta_{2}<1$, then for any solution $f \not \equiv 0$ of $(3)$, we have $\lambda(f) \geq n$.

It is natural to ask about the exponent of convergence of the zerosequence of solutions of the higher order linear differential equation (1). In the present paper we shall investigate this problem and obtain the following result which improves all the theorems mentioned earlier.

Theorem 1.1. Let $P_{1}(z), P_{2}(z), P_{3}(z)$ be as in Theorem $C$ and $Q_{1}(z)$, $Q_{2}(z), Q_{3}(z), a_{j}(z)(j=1, \ldots, k-1)$ be entire functions of order less than $n$ and $k \geq 2$.

(i) If $\zeta_{1} / \zeta_{2}$ is non-real and $0<\lambda=\zeta_{3} / \zeta_{2}<1 / k$, then for any solution $f \not \equiv 0$ of $(1)$, we have $\lambda(f)=\infty$.

(ii) If $0<\zeta_{1} / \zeta_{2}<1 / 2 k$ and $0<\lambda=\zeta_{3} / \zeta_{2}<1$, then for any solution $f \not \equiv 0$ of $(1)$, we have $\lambda(f) \geq n$.

2. Notations and some lemmas. To prove the theorem, we need some notations and a series of lemmas. Let $P_{j}(z)(j=1,2,3)$ be polynomials of degree $n \geq 1, P_{j}(z)=\left(\alpha_{j}+i \beta_{j}\right) z^{n}+\cdots, \alpha_{j}, \beta_{j} \in \mathbb{R}$. Define

$$
\begin{gathered}
\delta\left(P_{j}, \theta\right)=\delta_{j}(\theta)=\alpha_{j} \cos n \theta-\beta_{j} \sin n \theta, \quad \theta \in[0,2 \pi)(j=1,2,3), \\
S_{j}^{+}=\left\{\theta: \delta_{j}(\theta)>0\right\}, \quad S_{j}^{-}=\left\{\theta: \delta_{j}(\theta)<0\right\} \quad(j=1,2,3) .
\end{gathered}
$$

Let $f(z)$ and $a(z)$ be meromorphic functions in the plane that satisfy

$$
T(r, a)=o\{T(r, f)\},
$$

except possibly for a set of $r$ having finite linear measure. We then say that $a(z)$ is a small function with respect to $f(z)$.

LEMma 2.1 (4]). Let $f(z)$ be a transcendental meromorphic function with $\sigma(f)=\sigma<\infty$, and $\Gamma=\left\{\left(k_{1}, j_{1}\right), \ldots,\left(k_{m}, j_{m}\right)\right\}$ be a finite set of distinct pairs of integers which satisfy $k_{i}>j_{i} \geq 0$ for $i=1, \ldots, m$. Let $\varepsilon>0$ be a given constant. Then there exists a set $E \subset[0,2 \pi)$ of linear measure zero 
$\nu$ such that if $\varphi \in[0,2 \pi) \backslash E$ then there is a constant $R_{1}=R_{1}(\varphi)>1$ such that for all $z$ satisfying $\arg z=\varphi$ and $|z|=r>R_{1}$, and for all $(k, j) \in \Gamma$, we have

$$
\left|\frac{f^{(k)}(z)}{f^{(j)}(z)}\right| \leq|z|^{(k-j)(\sigma-1+\varepsilon)} .
$$

Lemma $2.2([2,9])$. Suppose that $P(z)=(\alpha+\beta i) z^{n}+\cdots(\alpha, \beta$ are real numbers, $|\alpha|+|\beta| \neq 0)$ is a polynomial of degree $n \geq 1$, and $A(z)$ $(\not \equiv 0)$ is an entire function with $\sigma(A)<n$. Set $g(z)=A(z) \overline{e^{P(z)}}, z=r e^{i \theta}$, $\delta(P, \theta)=\alpha \cos n \theta-\beta \sin n \theta$. Then for any given $\varepsilon>0$, there exists a set $H_{1} \subset[0,2 \pi)$ of linear measure zero such that for any $\theta \in[0,2 \pi) \backslash\left(H_{1} \cup H_{2}\right)$, where $H_{2}=\{\theta \in[0,2 \pi): \delta(P, \theta)=0\}$ is a finite set, there is $R>0$ such that for $|z|=r>R$, we have:

(i) If $\delta(P, \theta)>0$, then

$$
\exp \left\{(1-\varepsilon) \delta(P, \theta) r^{n}\right\}<\left|g\left(r e^{i \theta}\right)\right|<\exp \left\{(1+\varepsilon) \delta(P, \theta) r^{n}\right\} .
$$

(ii) If $\delta(P, \theta)<0$, then

$$
\exp \left\{(1+\varepsilon) \delta(P, \theta) r^{n}\right\}<\left|g\left(r e^{i \theta}\right)\right|<\exp \left\{(1-\varepsilon) \delta(P, \theta) r^{n}\right\} .
$$

LEMma $2.3([1])$. Suppose $\pi(z)$ is the canonical product formed with the zeros $\left\{z_{n}: n=1,2, \ldots,\right\}\left(z_{n} \neq 0\right)$ of an entire function $f(z)$. Set $O_{n}=\left\{z:\left|z-z_{n}\right|<\left|z_{n}\right|^{-\alpha}\right\}(\alpha(>\lambda(f))$ is a constant). Then for any given $\varepsilon>0$,

$$
|\pi(z)| \geq \exp \left\{-|z|^{\lambda(f)+\varepsilon}\right\} \quad \text { for } z \notin \bigcup_{n=1}^{\infty} O_{n} .
$$

LEMMA $2.4([3])$. Let $f(z)$ be an entire function of order $\sigma(f)=\alpha<$ $+\infty$. Then for any given $\varepsilon>0$, there is a set $E \subset[1, \infty)$ of finite linear measure and finite logarithmic measure such that for all z satisfying $|z| \notin$ $[0,1] \cup E$, we have

$$
\exp \left\{-r^{\alpha+\varepsilon}\right\} \leq|f(z)| \leq \exp \left\{r^{\alpha+\varepsilon}\right\}
$$

Lemma 2.5 ([10]). Let $P_{j}(z)(j=1,2,3)$ be polynomials of degree $n \geq 1$, $P_{1}(z)=\zeta z^{n}+B_{1}(z), \quad P_{2}(z)=\rho_{1} \zeta z^{n}+B_{2}(z), \quad P_{3}(z)=\rho_{2} \zeta z^{n}+B_{3}(z)$, where $\zeta=\alpha+i \beta, \alpha, \beta \in \mathbb{R},|\alpha|+|\beta| \neq 0,0<\rho_{1}<1,0<\rho_{2}<1$, and $B_{1}(z), B_{2}(z), B_{3}(z)$ are polynomials of degree at most $n-1$. Let $Q_{1}(z) \not \equiv 0$, $Q_{2}(z), Q_{3}(z)$ be entire functions of order less than $n$. Then for any given $\varepsilon>0$, there exist a set $E$ of finite linear measure and a constant $\xi(n-1<$ $\xi<n)$ such that

$m\left(r, Q_{1} e^{P_{1}}+Q_{2} e^{P_{2}}+Q_{3} e^{P_{3}}\right) \geq(1-\varepsilon) m\left(r, e^{P_{1}}\right)+O\left(r^{\xi}\right), \quad r \rightarrow \infty(r \notin E)$. 
LEMMA 2.6 ([11]). Let $f(z)$ be an entire function and write $f(z)=\pi e^{h}$. Then

(i) $\frac{f^{(k)}}{f}=\left(h^{\prime}\right)^{k}+k \frac{\pi^{\prime}}{\pi}\left(h^{\prime}\right)^{k-1}+\frac{k(k-1)}{2}\left(h^{\prime}\right)^{k-2} h^{\prime \prime}+H_{k-2}\left(h^{\prime}\right) \quad(k \geq 2)$,

where $H_{k-2}\left(h^{\prime}\right)$ is a differential polynomial of degree no more than $k-2$ in $h^{\prime}$, and its coefficients are terms of the type $c\left(\pi^{\prime} / \pi\right)^{s_{1}} \cdots\left(\pi^{(k)} / \pi\right)^{s_{k}}$, where $c$ is a constant and $s_{1}, \ldots, s_{k}$ are non-negative integers; and

$$
\frac{f^{(k+1)}}{f}-\frac{f^{(k)}}{f} \frac{f^{\prime}}{f}=k\left(h^{\prime}\right)^{k-1} h^{\prime \prime}+H_{k-1}\left(h^{\prime}\right) \quad(k \geq 1),
$$

where $H_{k-1}\left(h^{\prime}\right)$ is a differential polynomial of degree no more than $k-1$ in $h^{\prime}$, and its coefficients are terms of the type $c\left(\pi^{\prime} / \pi\right)^{s_{1}} \cdots\left(\pi^{(k+1)} / \pi\right)^{s_{k+1}}$, where $c$ is a constant and $s_{1}, \ldots, s_{k+1}$ are non-negative integers.

LEMMA $2.7([1])$. Let $U_{1}(z), h(z), Q_{1}(z), P_{1}(z)$ be entire functions satisfying $U_{1}=Q_{1} h^{\prime \prime}-\frac{1}{k}\left(Q_{1}^{\prime}+Q_{1} P_{1}^{\prime}\right) h^{\prime}$. Then

$$
Q_{1}^{n-1} h^{(n)}=A_{1, n-2}\left(U_{1}, Q_{1}\right)+B_{n-1}\left(Q_{1}\right) h^{\prime} \quad(n \geq 2),
$$

where $A_{1, n-2}\left(U_{1}, Q_{1}\right)$ is an algebraic expression in $U_{1}^{(j)}, Q_{1}^{(j)}, P_{1}^{(j)}(j=0,1$, $\ldots, l)$, involving addition, subtraction and multiplication, where the degree of $U_{1}^{(j)}$ is no more than 1 and the degree of $Q_{1}^{(j)}$ is no more than $l ; B_{d}\left(Q_{1}\right)$ is a differential polynomial of degree no more than $d$ in $Q_{1}$, its coefficients are algebraic expressions in $P_{1}^{(i)}(i=1, \ldots, d)$ and $1 / k$, involving addition, subtraction and multiplication.

LEMMA 2.8. Let $h(z)$ and $c_{j}(z)(j=0,1, \ldots, k-1)$ be meromorphic functions satisfying

$$
c_{k-1}(z)\left(h^{\prime}\right)^{k-1}+c_{k-2}(z)\left(h^{\prime}\right)^{k-2}+\cdots+c_{1}(z) h^{\prime}+c_{0}(z)=0 .
$$

Then

$$
m\left(r, h^{\prime}\right) \leq \sum_{j=0}^{k-1} T\left(r, c_{j}(z)\right)+O(1)
$$

LEMMA 2.9. Let $h$ be a meromorphic function of finite order, and $E_{k-1}\left(h^{\prime}\right)$ a differential polynomial of degree no more than $k-1$, whose coefficients are meromorphic functions $a_{j}(z)(j=0,1, \ldots, k-1)$ with $\sigma\left(a_{j}\right)<n$. Then for sufficiently large $r$,

$$
m\left(r,\left(h^{\prime}\right)^{k}+E_{k-1}\left(h^{\prime}\right)\right) \leq k m\left(r, h^{\prime}\right)+O\left(r^{\xi}\right)
$$

where $0<\max \left\{\sigma\left(a_{j}\right): j=0,1, \ldots, k-1\right\}<\xi<n$.

REMARK 2.1. Lemmas 2.8 and 2.9 are immediate consequences of the Valiron-Mohon'ko theorem (see [8]) and/or Clunie technique. 
3. Proof of Theorem 1.1. Since $\zeta_{3}=\lambda \zeta_{2}, \lambda>0$, we have $S_{2}^{+}=S_{3}^{+}$ and $S_{2}^{-}=S_{3}^{-}$. We see that $S_{j}^{+}$and $S_{j}^{-}$have $n$ components $S_{j k}^{+}$and $S_{j k}^{-}$ respectively $(j=1,2,3 ; k=1, \ldots, n)$. Hence we write

$$
S_{j}^{+}=\bigcup_{k=1}^{n} S_{j k}^{+}, \quad S_{j}^{-}=\bigcup_{k=1}^{n} S_{j k}^{-} \quad(j=1,2,3) .
$$

Furthermore, we define

$$
\begin{aligned}
& D_{12}=\left\{\theta \in S_{1}^{+} \cap S_{2}^{+}: \delta_{1}(\theta)>\frac{k(\lambda+1)}{k-1} \delta_{2}(\theta)\right\}, \\
& D_{21}=\left\{\theta \in S_{1}^{+} \cap S_{2}^{+}: \delta_{2}(\theta)>\frac{\lambda+1}{\lambda} \delta_{1}(\theta)\right\} .
\end{aligned}
$$

(i) Let $f \not \equiv 0$ be a solution of (1). Suppose that $\lambda(f)<\infty$. Write $f=\pi e^{h}$, where $\pi$ is the canonical product of the zeros of $f$, and $h$ is an entire function. From our hypothesis, we have $\sigma(\pi)=\lambda(\pi)<\infty$. From (1), we get

$$
\frac{f^{(k)}}{f}+a_{k-1} \frac{f^{(k-1)}}{f}+\cdots+a_{1} \frac{f^{\prime}}{f}+D(z)=0,
$$

By Lemma 2.6(i), we get

$$
\left(h^{\prime}\right)^{k}=E_{k-1}\left(h^{\prime}\right)-Q_{1}(z) e^{P_{1}(z)}-Q_{2}(z) e^{P_{2}(z)}-Q_{3}(z) e^{P_{3}(z)},
$$

where $E_{k-1}\left(h^{\prime}\right)$ is a differential polynomial of degree no more than $k-1$ in $h^{\prime}$, and its coefficients are terms of the type $c a_{j}^{p}(z)\left(\pi^{\prime} / \pi\right)^{s_{1}} \cdots\left(\pi^{(k)} / \pi\right)^{s_{k}}$ $(j=1, \ldots, k-1)$, where $c$ is a constant, $s_{1}, \ldots, s_{k}$ are non-negative integers and $p$ is 0 or 1 .

Eliminating $e^{P_{1}}$ from (4), we have

$$
\begin{aligned}
& Q_{1}\left(\frac{f^{(k+1)}}{f}-\frac{f^{(k)}}{f} \frac{f^{\prime}}{f}\right)+a_{k-1} Q_{1}\left(\frac{f^{(k)}}{f}-\frac{f^{(k-1)}}{f} \frac{f^{\prime}}{f}\right)+a_{1} Q_{1}\left(\frac{f^{\prime \prime}}{f}-\frac{f^{\prime}}{f} \frac{f^{\prime}}{f}\right) \\
& \quad-\left(Q_{1}^{\prime}+Q_{1} P_{1}^{\prime}\right)\left(\frac{f^{(k)}}{f}+a_{k-1} \frac{f^{(k-1)}}{f}+\cdots+a_{1} \frac{f^{\prime}}{f}+Q_{2} e^{P_{2}}+Q_{3} e^{P_{3}}\right) \\
& +Q_{1}\left[a_{k-1}^{\prime} \frac{f^{(k-1)}}{f}+\cdots+a_{1}^{\prime} \frac{f^{\prime}}{f}\right]+Q_{1}\left(Q_{2}^{\prime}+Q_{2} P_{2}^{\prime}\right) e^{P_{2}} \\
& +Q_{1}\left(Q_{3}^{\prime}+Q_{3} P_{3}^{\prime}\right) e^{P_{3}}=0 .
\end{aligned}
$$

By Lemma 2.6(ii), we can write this as

$$
\begin{aligned}
k U_{1}\left(h^{\prime}\right)^{k-1}= & F_{k-1}^{1}\left(h^{\prime}\right)+e^{P_{2}}\left[Q_{2}\left(Q_{1}^{\prime}+Q_{1} P_{1}^{\prime}\right)-Q_{1}\left(Q_{2}^{\prime}+Q_{2} P_{2}^{\prime}\right)\right] \\
& +e^{P_{3}}\left[Q_{3}\left(Q_{1}^{\prime}+Q_{1} P_{1}^{\prime}\right)-Q_{1}\left(Q_{3}^{\prime}+Q_{3} P_{3}^{\prime}\right)\right]
\end{aligned}
$$

where

$$
U_{1}=Q_{1} h^{\prime \prime}-\frac{1}{k}\left(Q_{1}^{\prime}+Q_{1} P_{1}^{\prime}\right) h^{\prime}
$$


and $F_{k-1}^{1}\left(h^{\prime}\right)$ is a differential polynomial of degree no more than $k-1$ in $h^{\prime}$, with coefficients of the type $c\left(a_{j}(z)\right)^{p}\left(a_{j}^{\prime}(z)\right)^{q}\left(Q_{1}\right)^{l}\left(Q_{1}^{\prime}\right)^{t}\left(P_{1}^{\prime}\right)^{u}\left(\pi^{\prime} / \pi\right)^{s_{1}} \ldots$ $\left(\pi^{(k)} / \pi\right)^{s_{k}}$, where $c$ is a constant, $s_{1}, \ldots, s_{k}$ are non-negative integers and each of $p, q, l, t, u$ is 0 or 1 . Similarly, we obtain

$$
\begin{aligned}
k U_{2}\left(h^{\prime}\right)^{k-1}= & F_{k-1}^{2}\left(h^{\prime}\right)+e^{P_{1}}\left[Q_{1}\left(Q_{2}^{\prime}+Q_{2} P_{2}^{\prime}\right)-Q_{2}\left(Q_{1}^{\prime}+Q_{1} P_{1}^{\prime}\right)\right] \\
& +e^{P_{3}}\left[Q_{3}\left(Q_{2}^{\prime}+Q_{2} P_{2}^{\prime}\right)-Q_{2}\left(Q_{3}^{\prime}+Q_{3} P_{3}^{\prime}\right)\right],
\end{aligned}
$$

where

$$
U_{2}=Q_{2} h^{\prime \prime}-\frac{1}{k}\left(Q_{2}^{\prime}+Q_{2} P_{2}^{\prime}\right) h^{\prime},
$$

and $F_{k-1}^{2}\left(h^{\prime}\right)$ is a differential polynomial of degree no more than $k-1$ in $h^{\prime}$, with coefficients of the type $c\left(a_{j}(z)\right)^{p}\left(a_{j}^{\prime}(z)\right)^{q}\left(Q_{2}\right)^{l}\left(Q_{2}^{\prime}\right)^{t}\left(P_{2}^{\prime}\right)^{u}\left(\pi^{\prime} / \pi\right)^{s_{1}}$ $\cdots\left(\pi^{(k)} / \pi\right)^{s_{k}}$, where $c$ is a constant, $s_{1}, \ldots, s_{k}$ are non-negative integers and each of $p, q, l, t, u$ is 0 or 1 .

Let $\max \left\{\sigma\left(Q_{i}\right), \sigma\left(a_{j}\right): i=1,2,3 ; j=1, \ldots, k-1\right\}<\xi_{1}<\xi_{2}<\xi_{3}<n$. From Lemma 2.4 we get

$$
\mid Q_{i}\left(r e^{i \theta}\left|\leq \exp \left(r^{\xi_{1}}\right) \quad(i=1,2,3), \quad\right| a_{j}(z) \mid \leq \exp \left(r^{\xi_{1}}\right) \quad(j=1, \ldots, k-1),\right.
$$

for sufficiently large $r$ and for any $\theta \in[0,2 \pi)$. Applying the Clunie Lemma [5. Lemma 3.3] to (5), for any given $\varepsilon>0$ we get

$$
\begin{aligned}
T\left(r, h^{\prime}\right)= & m\left(r, h^{\prime}\right) \\
\leq & m\left(r, Q_{1} e^{P_{1}}+Q_{2} e^{P_{2}}+Q_{3} e^{P_{3}}\right) \\
& +O\left(\sum_{j=1}^{k} m\left(r, \pi^{(j)} / \pi\right)+\sum_{i=1}^{k-1} m\left(r, a_{i}\right)\right)+S\left(r, h^{\prime}\right) \\
\leq & O\left(r^{n+\varepsilon}\right)+S\left(r, h^{\prime}\right),
\end{aligned}
$$

which implies $\sigma\left(h^{\prime}\right) \leq n$. It follows from (7) and (9) that $\sigma\left(U_{1}\right) \leq n$ and $\sigma\left(U_{2}\right) \leq n$ respectively.

In the following, we will show that there exists a set $E_{0} \subset[0,2 \pi)$ with $m\left(E_{0}\right)=0$ such that if $\theta \in S_{2}^{-} \backslash E_{0}$, then

$$
\left|U_{1}\left(r e^{i \theta}\right)\right| \leq O\left(\exp \left\{r^{\xi_{2}}\right\}\right), \quad r \rightarrow \infty .
$$

If $\left|h^{\prime}\left(r e^{i \theta}\right)\right| \leq 1$, from Lemmas 2.1, 2.2 and 2.4 and (7), we have

$$
\begin{aligned}
\left|U_{1}\left(r e^{i \theta}\right)\right| \leq & \frac{\left|h^{\prime \prime}\left(r e^{i \theta}\right)\right|}{\left|h^{\prime}\left(r e^{i \theta}\right)\right|}\left|Q_{1}\left(r e^{i \theta}\right)\right|+\frac{1}{k}\left|P_{1}^{\prime}\left(r e^{i \theta}\right)\right|\left|Q_{1}\left(r e^{i \theta}\right)\right| \\
& +\frac{1}{k} \frac{\left|Q_{1}^{\prime}\left(r e^{i \theta}\right)\right|}{\left|Q_{1}\left(r e^{i \theta}\right)\right|}\left|Q_{1}\left(r e^{i \theta}\right)\right| \\
\leq & O\left(\exp \left\{r^{\xi_{2}}\right\}\right), \quad r \rightarrow \infty .
\end{aligned}
$$


Assume $\left|h^{\prime}\left(r e^{i \theta}\right)\right| \geq 1$. Since $F_{k-1}^{1}\left(h^{\prime}\right)$ is the sum of a finite number of terms of the type

$$
\begin{aligned}
H(z)= & c\left(a_{j}(z)\right)^{p}\left(a_{j}^{\prime}(z)\right)^{q}\left(Q_{1}\right)^{l}\left(Q_{1}^{\prime}\right)^{t}\left(P_{1}^{\prime}\right)^{u}\left(\frac{\pi^{\prime}}{\pi}\right)^{s_{1}} \cdots\left(\frac{\pi^{(k)}}{\pi}\right)^{s_{k}} \\
& \times\left(h^{\prime}\right)^{l_{0}}\left(h^{\prime \prime}\right)^{l_{1}} \cdots\left(h^{(v)}\right)^{l_{v-1}},
\end{aligned}
$$

where $l_{0}, l_{1}, \ldots, l_{v-1}$ are non-negative integers and $l_{0}+l_{1}+\cdots+l_{v-1} \leq k-1$, from Lemma 2.1 we get

$$
\begin{aligned}
& \frac{\left|H\left(r e^{i \theta}\right)\right|}{\left|h^{\prime}\left(r e^{i \theta}\right)\right|^{k-1}} \\
& \leq|c|\left|a_{j}\left(r e^{i \theta}\right)\right|^{p}\left|a_{j}^{\prime}\left(r e^{i \theta}\right)\right|^{q}\left|Q_{1}\left(r e^{i \theta}\right)\right|^{l}\left|Q_{1}^{\prime}\left(r e^{i \theta}\right)\right|^{t}\left|P_{1}^{\prime}\left(r e^{i \theta}\right)\right|^{u} \\
& \quad \times\left|\frac{\pi^{\prime}\left(r e^{i \theta}\right)}{\pi\left(r e^{i \theta}\right)}\right|^{s_{1}} \cdots\left|\frac{\pi^{(k)}\left(r e^{i \theta}\right)}{\pi\left(r e^{i \theta}\right)}\right|^{s_{k}} \frac{\left|h^{\prime \prime}\left(r e^{i \theta}\right)\right|^{l_{1}}}{\left|h^{\prime}\left(r e^{i \theta}\right)\right|} \cdots \frac{\left|h^{(v)}\left(r e^{i \theta}\right)\right|^{l_{v-1}}}{\left|h^{\prime}\left(r e^{i \theta}\right)\right|} \\
& \leq
\end{aligned}
$$

Thus

$$
\frac{\left|F_{k-1}^{1}\left(r e^{i \theta}\right)\right|}{\left|h^{\prime}\left(r e^{i \theta}\right)\right|^{k-1}} \leq O\left(\exp \left\{r^{\xi_{2}}\right\}\right)
$$

From (8), (13) and Lemma 2.2, we get

$$
\begin{aligned}
& k\left|U_{1}\left(r e^{i \theta}\right)\right| \\
\leq & \frac{\left|F_{k-1}^{1}\left(r e^{i \theta}\right)\right|}{\left|h^{\prime}\left(r e^{i \theta}\right)\right|^{k-1}}+\left|e^{P_{2}\left(r e^{i \theta}\right)}\right| \mid Q_{2}\left(r e^{i \theta}\right)\left(Q_{1}^{\prime}\left(r e^{i \theta}\right)+Q_{1}\left(r e^{i \theta}\right) P_{1}^{\prime}\left(r e^{i \theta}\right)\right) \\
& -Q_{1}\left(r e^{i \theta}\right)\left(\left(Q_{2}^{\prime}\left(r e^{i \theta}\right)+Q_{2}\left(r e^{i \theta}\right) P_{2}^{\prime}\left(r e^{i \theta}\right)\right) \mid\right. \\
& +\left|e^{P_{3}\left(r e^{i \theta}\right)}\right| \mid Q_{3}\left(r e^{i \theta}\right)\left(Q_{1}^{\prime}\left(r e^{i \theta}\right)+Q_{1}\left(r e^{i \theta}\right) P_{1}^{\prime}\left(r e^{i \theta}\right)\right) \\
& -Q_{1}\left(r e^{i \theta}\right)\left(Q_{3}^{\prime}\left(r e^{i \theta}\right)+Q_{3}\left(r e^{i \theta}\right) P_{3}^{\prime}\left(r e^{i \theta}\right) \mid\right. \\
\leq & O\left(\exp \left\{r^{\xi_{2}}\right\}\right), \quad r \rightarrow \infty .
\end{aligned}
$$

From (11) and (14), we obtain (10).

We note that there exist $\bar{\theta}_{j}(j=1,2,3)$ satisfying $\delta_{j}(\theta)=0$ on the rays $\arg z=\bar{\theta}_{j}+q \pi / n$, where $q=0, \ldots, 2 n-1$, which form $2 n$ sectors of opening $\pi / n$ each, thus we may assume that $\bar{\theta}_{j} \in[0, \pi / n)$. Since $\zeta_{2}=\lambda \zeta_{3}, \lambda>0$, we have $\bar{\theta}_{2}=\bar{\theta}_{3}$. Write $\bar{\theta}_{j q}=\bar{\theta}_{j}+q \pi / n, j=1,2$. If there are some integers $q_{1}$ and $q_{2}$ such that $\bar{\theta}_{1 q_{1}}=\bar{\theta}_{2 q_{2}}$, then $\bar{\theta}_{1}-\bar{\theta}_{2}+\left(q_{1}-q_{2}\right) \pi / n=0$, and we have $\tan n \bar{\theta}_{j}=\alpha_{j} / \beta_{j}, j=1,2$. This gives

$$
0=\tan \left(n \bar{\theta}_{1}-n \bar{\theta}_{2}+\left(q_{1}-q_{2}\right) \pi\right)=\frac{\alpha_{1} \beta_{2}-\alpha_{2} \beta_{1}}{\alpha_{1} \alpha_{2}+\beta_{1} \beta_{2}} .
$$

This contradicts the assumption that $\zeta_{1} / \zeta_{2}$ is non-real. Hence each component of $S_{1}^{+}$and $S_{2}^{+}$contains a component of $S_{1}^{+} \cap S_{2}^{+}$. The boundaries of the 
components of $S_{1}^{+} \cap S_{2}^{+}$are some of the rays $\arg z=\bar{\theta}_{j q}$. We fix a component of $S_{1}^{+} \cap S_{2}^{+}$, say $S^{*}$. We may write

$$
S^{*}=\left\{\theta \in S_{1}^{+} \cap S_{2}^{+}: \theta_{1}^{*}<\theta<\theta_{2}^{*}, \delta_{1}\left(\theta_{1}^{*}\right)=\delta_{2}\left(\theta_{2}^{*}\right)=0\right\}
$$

or

$$
S^{*}=\left\{\theta \in S_{1}^{+} \cap S_{2}^{+}: \theta_{2}^{*}<\theta<\theta_{1}^{*}, \delta_{1}\left(\theta_{1}^{*}\right)=\delta_{2}\left(\theta_{2}^{*}\right)=0\right\} .
$$

Since every component of $S_{1}^{+}$and $S_{2}^{+}$has opening $\pi / n$, the rays $\arg z=\theta_{1}^{*}$ and $\arg z=\theta_{2}^{*}$ are contained in $S_{2}^{+}$and $S_{1}^{+}$respectively. We handle the first case, the proof of the second being similar. Then there exist $\eta_{1}, \eta_{2}>0$ such that

$$
\left\{\theta: \theta_{1}^{*}<\theta<\theta_{1}^{*}+\eta_{1}\right\} \subset D_{21}, \quad\left\{\theta: \theta_{2}^{*}-\eta_{2}<\theta<\theta_{2}^{*}\right\} \subset D_{12} .
$$

Hence there exists a $\theta \in\left(S_{2 k}^{+} \cap D_{12}\right) \backslash E_{0}$ for any $k=1, \ldots, n$. Take $0<$ $\frac{k(\lambda+1)}{k-1} \delta_{2}<\sigma_{2}<\sigma_{1}<\delta_{1}, 0<\varepsilon_{1}<1-\frac{\sigma_{1}}{\delta_{1}}, 0<\varepsilon_{2}<\frac{(k-1) \sigma_{2}}{k \delta_{2}}-1,0<\varepsilon_{3}<$ $\frac{(k-1) \sigma_{2}}{k \lambda \delta_{2}}-1$. By Lemma 2.2, we have

$$
\begin{aligned}
& \left|Q_{1} e^{P_{1}\left(r e^{i \theta}\right)}+Q_{2} e^{P_{2}\left(r e^{i \theta}\right)}+Q_{3} e^{P_{3}\left(r e^{i \theta}\right)}\right| \\
& \quad \geq\left|Q_{1} e^{P_{1}\left(r e^{i \theta}\right)}\right||1-| \frac{Q_{2}}{Q_{1}} e^{P_{2}\left(r e^{i \theta}\right)-P_{1}\left(r e^{i \theta}\right)}|-| \frac{Q_{3}}{Q_{1}} e^{P_{3}\left(r e^{i \theta}\right)-P_{1}\left(r e^{i \theta}\right)}|| \\
& \quad \geq \exp \left\{\left(1-\varepsilon_{1}\right) \delta_{1} r^{n}\right\}(1-o(1)) \\
& \quad \geq \exp \left\{\sigma_{1} r^{n}\right\}(1-o(1)), \quad r \rightarrow \infty .
\end{aligned}
$$

We assume that there exists an unbounded sequence $\left\{r_{q}\right\}$ such that $0<$ $\left|h^{\prime}\left(r_{q} e^{i \theta}\right)\right| \leq 1$. From (5), (15) and Lemma 2.1, we get

$$
\begin{aligned}
\exp \left\{\sigma_{1} r_{q}^{n}\right\}(1-o(1)) \leq & \left|h^{\prime}\left(r_{q} e^{i \theta}\right)\right|^{k}+\left|E_{k-1}\left(h^{\prime}\left(r_{q} e^{i \theta}\right)\right)\right| \\
\leq & 1+\sum|c|\left|a_{j}\left(r_{q} e^{i \theta}\right)\right|^{p}\left|\frac{\pi^{\prime}\left(r_{q} e^{i \theta}\right)}{\pi\left(r_{q} e^{i \theta}\right)}\right|^{s_{1}} \cdots\left|\frac{\pi^{(k)}\left(r_{q} e^{i \theta}\right)}{\pi\left(r_{q} e^{i \theta}\right)}\right|^{s_{k}} \\
& \times\left|h^{\prime}\left(r_{q} e^{i \theta}\right)\right|^{l_{0}} \cdots\left|h^{(v)}\left(r_{q} e^{i \theta}\right)\right|^{l_{v-1}} \\
\leq & 1+\sum|c|\left|a_{j}\left(r_{q} e^{i \theta}\right)\right|^{p}\left|\frac{\pi^{\prime}\left(r_{q} e^{i \theta}\right)}{\pi\left(r_{q} e^{i \theta}\right)}\right|^{s_{1}} \cdots\left|\frac{\pi^{(k)}\left(r_{q} e^{i \theta}\right)}{\pi\left(r_{q} e^{i \theta}\right)}\right|^{s_{k}} \\
& \times\left|\frac{h^{\prime \prime}\left(r_{q} e^{i \theta}\right)}{h^{\prime}\left(r_{q} e^{i \theta}\right)}\right|^{l_{1}} \cdots\left|\frac{h^{(v)}\left(r_{q} e^{i \theta}\right)}{h^{\prime}\left(r_{q} e^{i \theta}\right)}\right|^{l_{v-1}} \\
\leq & O\left(\exp \left\{r_{q}^{\xi_{2}}\right\}\right) \quad(q \rightarrow \infty),
\end{aligned}
$$

which is not true. Hence we may assume that $\left|h^{\prime}\left(r e^{i \theta}\right)\right| \geq 1$ for all $r$ suffi- 
ciently large. From (5), (15) and Lemma 2.2, we get

$$
\begin{aligned}
\exp \left\{\sigma_{1} r_{q}^{n}\right\}(1-o(1)) \leq & \left|h^{\prime}\left(r_{q} e^{i \theta}\right)\right|^{k}+\left|E_{k-1}\left(h^{\prime}\left(r_{q} e^{i \theta}\right)\right)\right| \\
\leq & \left|h^{\prime}\left(r_{q} e^{i \theta}\right)\right|^{k}\left[1+\sum|c|\left|a_{j}\left(r_{q} e^{i \theta}\right)\right|^{p}\left|\frac{\pi^{\prime}\left(r_{q} e^{i \theta}\right)}{\pi\left(r_{q} e^{i \theta}\right)}\right|^{s_{1}}\right. \\
& \left.\cdots\left|\frac{\pi^{(k)}\left(r_{q} e^{i \theta}\right)}{\pi\left(r_{q} e^{i \theta}\right)}\right|^{s_{k}}\left|\frac{h^{\prime \prime}\left(r_{q} e^{i \theta}\right)}{h^{\prime}\left(r_{q} e^{i \theta}\right)}\right|^{l_{1}} \cdots\left|\frac{h^{(v)}\left(r_{q} e^{i \theta}\right)}{h^{\prime}\left(r_{q} e^{i \theta}\right)}\right|^{l_{v-1}}\right] \\
\leq & \left|h^{\prime}\left(r_{q} e^{i \theta}\right)\right|^{k}\left(1+O\left(\exp \left\{r_{q}^{\xi_{2}}\right\}\right)\right) \quad(q \rightarrow \infty),
\end{aligned}
$$

i.e.

$$
\left|h^{\prime}\left(r e^{i \theta}\right)\right|^{k} \geq \frac{1-o(1)}{1+O\left(\exp \left\{r \xi_{2}\right\}\right)} \exp \left\{\sigma_{1} r^{n}\right\} \quad(r \rightarrow \infty) .
$$

Then we obtain for all $r$ large enough

$$
\left|h^{\prime}\left(r e^{i \theta}\right)\right| \geq \exp \left\{\frac{1}{k} \sigma_{2} r^{n}\right\} .
$$

From Lemma 2.1, (6) and (16), we get

$$
\begin{aligned}
\leq & \frac{\left|F_{k-1}^{1}\left(r e^{i \theta}\right)\right|}{\left|h^{\prime}\left(r e^{i \theta}\right)\right|^{k-1}} \\
& +\frac{\left|e^{P_{2}\left(r e^{i \theta}\right)}\right|}{\left|h^{\prime}\left(r e^{i \theta}\right)\right|^{k-1}}\left[\left|Q_{2}\left(r e^{i \theta}\right)\right|\left(\frac{\mid Q_{1}^{\prime}\left(r e^{i \theta} \mid\right.}{\left|Q_{1}\left(r e^{i \theta}\right)\right|}\left|Q_{1}\left(r e^{i \theta}\right)\right|+\left|Q_{1}\left(r e^{i \theta}\right)\right|\left|P_{1}^{\prime}\left(r e^{i \theta}\right)\right|\right)\right. \\
& \left.+\left|Q_{1}\left(r e^{i \theta}\right)\right|\left(\frac{\left|Q_{2}^{\prime}\left(r e^{i \theta}\right)\right|}{\left|Q_{2}\left(r e^{i \theta}\right)\right|}\left|Q_{2}\left(r e^{i \theta}\right)\right|+\left|Q_{2}\left(r e^{i \theta}\right)\right|\left|P_{2}^{\prime}\left(r e^{i \theta}\right)\right|\right)\right] \\
& +\frac{\left|e^{P_{3}\left(r e^{i \theta}\right)}\right|}{\left|h^{\prime}\left(r e^{i \theta}\right)\right|^{k-1}}\left[\left|Q_{3}\left(r e^{i \theta}\right)\right|\left(\frac{\left|Q_{1}^{\prime}\left(r e^{i \theta}\right)\right|}{\left|Q_{1}\left(r e^{i \theta}\right)\right|}\left|Q_{1}\left(r e^{i \theta}\right)\right|+\left|Q_{1}\left(r e^{i \theta}\right)\right|\left|P_{1}^{\prime}\left(r e^{i \theta}\right)\right|\right)\right. \\
& \left.+\left|Q_{1}\left(r e^{i \theta}\right)\right|\left(\frac{\left|Q_{3}^{\prime}\left(r e^{i \theta}\right)\right|}{\left|Q_{3}\left(r e^{i \theta}\right)\right|}+\left|Q_{3}\left(r e^{i \theta}\right)\right|\left|P_{3}^{\prime}\left(r e^{i \theta}\right)\right|\right)\right] \\
\leq & O\left(\exp \left\{r r^{\xi_{2}}\right\}\right)+(1+o(1)) \exp \left\{\left(\delta_{2}\left(1+\varepsilon_{2}\right)-\frac{(k-1) \sigma_{2}}{k}\right) r^{n}\right\} \\
& +(1+o(1)) \exp \left\{\left(\lambda \delta_{2}\left(1+\varepsilon_{3}\right)-\frac{(k-1) \sigma_{2}}{k}\right) r^{n}\right\} \quad(r \rightarrow \infty) .
\end{aligned}
$$

Since $\delta_{2}\left(1+\varepsilon_{2}\right)-(k-1) \sigma_{2} / k<0$ and $\lambda \delta_{2}\left(1+\varepsilon_{3}\right)-(k-1) \sigma_{2} / k<0$, this gives that for all sufficiently large $r$,

$$
\left|U_{1}\left(r e^{i \theta}\right)\right| \leq O\left(\exp \left\{r^{\xi_{2}}\right\}\right) .
$$

Now we fix a $\gamma\left(=\gamma_{2 k}\right) \in\left(S_{2 k}^{+} \cap D_{12}\right) \backslash E_{0}, k=1, \ldots, n$. Then we find $\gamma_{1}, \gamma_{2} \in S_{2}^{-} \backslash E_{0}$ with $\gamma_{1}<\gamma<\gamma_{2}$ such that $\gamma-\gamma_{1}<\pi / n$ and $\gamma_{2}-\gamma<\pi / n$. 
We first prove that for any $\theta$ with $\gamma_{1} \leq \theta \leq \gamma$, we have

$$
\left|U_{1}\left(r e^{i \theta}\right)\right| \leq O\left(\exp \left\{r^{\xi_{3}}\right\}\right) \quad(r \rightarrow \infty) .
$$

Write $\gamma-\gamma_{1}=\pi /\left(n+\tau_{1}\right)$ with $\tau_{1}>0$. Since $\sigma\left(U_{1}\right) \leq n$, we have $\left|U_{1}\left(r e^{i \theta}\right)\right| \leq$ $e^{r^{n+\tau_{2}}}$ with $0<\tau_{2}<\tau_{1}$ for sufficiently large $r$. Set

$$
g(z)=U_{1}(z) / \exp \left(\left(z e^{-\left(\gamma+\gamma_{1}\right) / 2}\right)^{\xi_{3}}\right) .
$$

Then $g(z)$ is regular in the region $\left\{z: \gamma_{1} \leq \arg z \leq \gamma\right\}$. Since $\gamma_{1} \leq \arg z=$ $\theta \leq \gamma$ and $\gamma-\gamma_{1}<\pi / n$, we infer that $\cos \arg \left(\left(z e^{-\left(\gamma+\gamma_{1}\right) / 2}\right)^{\xi_{3}}\right) \geq K$ for some $K>0$. In fact,

$$
-\frac{\pi}{2}<-\frac{\pi \xi_{3}}{2 n} \leq-\xi_{3} \frac{\gamma-\gamma_{1}}{2} \leq \arg \left(\left(z e^{-\left(\gamma+\gamma_{1}\right) / 2}\right)^{\xi_{3}}\right) \leq \xi_{3} \frac{\gamma-\gamma_{1}}{2} \leq \frac{\pi \xi_{3}}{2 n}<\frac{\pi}{2} .
$$

Hence for $\gamma_{1}<\theta<\gamma$,

$$
\left|g\left(r e^{i \theta}\right)\right| \leq\left|\frac{U_{1}\left(r e^{i \theta}\right)}{\exp \left\{K r^{\xi_{3}}\right\}}\right| \leq O\left(\exp \left\{r^{n+\tau_{2}}\right\}\right) \quad(r \rightarrow \infty) .
$$

It follows from (10) and (18) that for some $M>0$, as $r \rightarrow \infty$,

$$
\left|g\left(r e^{i \gamma_{1}}\right)\right| \leq \frac{O\left(e^{r \xi_{2}}\right)}{\exp \left\{K r^{\xi_{3}}\right\}} \leq M
$$

and

$$
\left|g\left(r e^{i \gamma}\right)\right| \leq \frac{O\left(e^{r^{\xi_{2}}}\right)}{\exp \left\{K r^{\xi_{3}}\right\}} \leq M
$$

By the Phragmén-Lindelöf theorem, we obtain (19). Similarly we see that (19) holds for $\gamma<\theta<\gamma_{2}$. Hence we conclude that (19) holds for any $\theta \in[0,2 \pi)$.

By a similar proof as before we can prove that for any $\theta \in[0,2 \pi)$,

$$
\left|U_{2}\left(r e^{i \theta}\right)\right| \leq O\left(\exp \left\{r^{\xi_{3}}\right\}\right) \quad(r \rightarrow \infty) .
$$

By (7) and (9), we have

$$
Q_{2} U_{1}-Q_{1} U_{2}=\frac{1}{k} h^{\prime}\left[Q_{1}\left(Q_{2}^{\prime}+Q_{2} P_{2}^{\prime}\right)-Q_{2}\left(Q_{1}^{\prime}+Q_{1} P_{1}^{\prime}\right)\right] .
$$

Since $\sigma\left(Q_{j}\right)<\xi_{2}<\xi_{3}(j=1,2,3)$, by (5), (10), (20) and Lemma 2.9,

$$
\begin{aligned}
m\left(r, Q_{1} e^{P_{1}(z)}\right. & \left.+Q_{2} e^{P_{2}(z)}+Q_{3} e^{P_{3}(z)}\right) \\
& \leq k m\left(r, h^{\prime}\right)+O(\log r) \\
& \leq k m\left(r, U_{1}-U_{2}\right)+O(\log r) \leq O\left(r^{\xi_{3}}\right) \quad(r \rightarrow \infty) .
\end{aligned}
$$

Since $\zeta_{1} / \zeta_{2}$ is non-real, $S_{1}^{+} \cap S_{2}^{-}$contains an interval $I=\left[\varphi_{1}, \varphi_{2}\right]$ satisfying $\min _{\theta \in I} \delta_{1}(\theta)=s>0$. By Lemma 2.2 , there exists an $R(I)(>0)$ such 
that for any $\theta \in I$ and $r \geq R(I)$,

$$
\begin{aligned}
& \left|Q_{1} e^{P_{1}\left(r e^{i \theta}\right)}\right| \geq \exp \left((1-\varepsilon) \delta_{1} r^{n}\right), \\
& \left|Q_{2} e^{P_{2}\left(r e^{i \theta}\right)}\right| \leq \exp \left((1-\varepsilon) \delta_{2} r^{n}\right), \\
& \left|Q_{3} e^{P_{3}\left(r e^{i \theta}\right)}\right| \leq \exp \left((1-\varepsilon) \lambda \delta_{2} r^{n}\right) .
\end{aligned}
$$

Hence, we have

$$
\begin{aligned}
m\left(r, Q_{1} e^{P_{1}(z)}+Q_{2} e^{P_{2}(z)}+Q_{3} e^{P_{3}(z)}\right) & \\
& \geq \int_{\varphi_{1}}^{\varphi_{2}} \log ^{+}\left|Q_{1} e^{P_{1}(z)}+Q_{2} e^{P_{2}(z)}+Q_{3} e^{P_{3}(z)}\right| d \theta \\
& \geq \int_{\varphi_{1}}^{\varphi_{2}}(1-o(1)) \log ^{+}\left|Q_{1} e^{P_{1}(z)}\right| d \theta \\
& \geq \int_{\varphi_{1}}^{\varphi_{1}}(1-o(1))(1-\varepsilon) s r^{n} d \theta \\
& \geq(1-o(1))(1-\varepsilon) s r^{n}\left(\varphi_{2}-\varphi_{1}\right) \quad(r \rightarrow \infty) .
\end{aligned}
$$

Combining (22) and (23) and recalling that $\xi_{3}<n$, we get a contradiction. Hence, $\lambda(f)=\infty$.

(ii) Let $f \not \equiv 0$ be a solution of (1). Write $f=\pi e^{h}$, suppose that $\lambda(f)<n$. From our hypothesis, we have $\sigma(\pi)=\lambda(\pi)<n$. Eliminating $e^{P_{1}}$ from (5), we have

$$
\begin{aligned}
k U\left(h^{\prime}\right)^{k-1}= & F_{k-1}\left(h^{\prime}\right)+e^{P_{2}}\left[Q_{2}\left(Q_{1}^{\prime}+Q_{1} P_{1}^{\prime}\right)-Q_{1}\left(Q_{2}^{\prime}+Q_{2} P_{2}^{\prime}\right)\right] \\
& +e^{P_{3}}\left[Q_{3}\left(Q_{1}^{\prime}+Q_{1} P_{1}^{\prime}\right)-Q_{1}\left(Q_{3}^{\prime}+Q_{3} P_{3}^{\prime}\right)\right]
\end{aligned}
$$

where

$$
U=Q_{1} h^{\prime \prime}-\frac{1}{k}\left(Q_{1}^{\prime}+Q_{1} P_{1}^{\prime}\right) h^{\prime},
$$

From (24), (25) and Lemma 2.7, we have

$$
\begin{aligned}
c_{k-1}(z)\left(h^{\prime}\right)^{k-1}+ & c_{k-2}\left(h^{\prime}\right)^{k-2}+\cdots+c_{1}(z) h^{\prime} \\
= & c_{0}(z)+e^{P_{2}}\left[Q_{2}\left(Q_{1}^{\prime}+Q_{1} P_{1}^{\prime}\right)-Q_{1}\left(Q_{2}^{\prime}+Q_{2} P_{2}^{\prime}\right)\right] \\
& +e^{P_{3}}\left[Q_{3}\left(Q_{1}^{\prime}+Q_{1} P_{1}^{\prime}\right)-Q_{1}\left(Q_{3}^{\prime}+Q_{3} P_{3}^{\prime}\right)\right],
\end{aligned}
$$

where $c_{j}(z)(j=0,1, \ldots, k-1)$ is an algebraic expression in $U^{(l)}(l=$ $0,1, \ldots, k-2), Q_{1}^{(i)}(i=0,1 \ldots, k-1), P_{1}^{(s)}(s=0,1, \ldots, l-1), 1 / k, 1 / Q_{1}$ and $a_{j}, a_{j}^{\prime}(j=1, \ldots, k-1)$, involving addition, subtraction and multiplication.

Now we suppose that at least one of $c_{j}(z)(j=1, \ldots, k-1)$ is not identically vanishing and the right hand side of $(26)$ does not vanish identically. Without loss of generality, suppose $c_{k-1}(z) \not \equiv 0$. Then from (26) and 
Lemma 2.8, we have

$$
\begin{aligned}
& T\left(r, h^{\prime}\right)=m\left(r, h^{\prime}\right) \\
& \quad \leq \sum_{i=0}^{k-1} T\left(r, c_{i}(z)\right)+m\left(r, e^{P_{2}}\left[Q_{2}\left(Q_{1}^{\prime}+Q_{1} P_{1}^{\prime}\right)-Q_{1}\left(Q_{2}^{\prime}+Q_{2} P_{2}^{\prime}\right)\right]\right. \\
& \left.\quad+e^{P_{3}}\left[Q_{3}\left(Q_{1}^{\prime}+Q_{1} P_{1}^{\prime}\right)-Q_{1}\left(Q_{3}^{\prime}+Q_{3} P_{3}^{\prime}\right)\right]\right)+O(1) .
\end{aligned}
$$

Take $\max \left\{\sigma\left(Q_{1}\right), \sigma\left(Q_{2}\right), \sigma\left(Q_{3}\right), \lambda(f)\right\}<\xi_{2}<\xi_{3}<n$. From (5), we obtain

$$
T\left(r, Q_{1} e^{P_{1}(z)}+Q_{2} e^{P_{2}(z)}+Q_{3} e^{P_{3}(z)}\right) \leq k T\left(r, h^{\prime}\right)+O(\log r) .
$$

By Lemma 2.5, we have

$$
\begin{aligned}
m\left(r, Q_{1} e^{P_{1}(z)}+Q_{2} e^{P_{2}(z)}+Q_{3} e^{P_{3}(z)}\right) & \\
& \geq(1-\varepsilon) m\left(r, e^{P_{1}}\right)+O\left(r^{\xi_{3}}\right) \quad(r \rightarrow \infty, r \notin E) .
\end{aligned}
$$

where $E$ has finite linear measure. From (28) and (29), we obtain

$$
T\left(r, h^{\prime}\right) \geq \frac{1-\varepsilon}{k} T\left(r, e^{P_{1}}\right)+O\left(r^{\xi_{3}}\right) \quad(r \rightarrow \infty, r \notin E) .
$$

Since $0<\rho=\zeta_{2} / \zeta_{1}<1 / 2 k, \zeta_{3}=\lambda \zeta_{2}, 0<\lambda<1$, we get

$\delta\left(P_{2}, \theta\right)=\rho \delta\left(P_{1}, \theta\right), \quad S_{1 k}^{+}=S_{2 k}^{+}=S_{3 k}^{+}, \quad S_{1 k}^{-}=S_{2 k}^{-}=S_{3 k}^{-} \quad(k=1, \ldots, n)$.

By the same reasoning as in (11) and (14), we have

$$
\left|U\left(r e^{i \theta}\right)\right| \leq O\left(\exp \left\{r^{\xi_{2}}\right\}\right) \quad(r \rightarrow \infty)
$$

for any $\theta \in S_{1}^{-} \backslash E_{0}, m\left(E_{0}\right)=0$. Also by the same reasoning as in (15)-(18), we have

$$
\left|U\left(r e^{i \theta}\right)\right| \leq O\left(\exp \left\{r^{\xi_{2}}\right\}\right) \quad(r \rightarrow \infty)
$$

for any $\theta \in S_{1}^{+} \backslash E_{0}, m\left(E_{0}\right)=0$. Since $\sigma(U) \leq n$, by the Phragmén-Lindelöf theorem, we have

$$
\left|U\left(r e^{i \theta}\right)\right| \leq O\left(\exp \left\{r^{\xi_{3}}\right\}\right) \quad(r \rightarrow \infty)
$$

for any $\theta \in[0,2 \pi)$.

In the following, we estimate $T\left(r, c_{j}\right)$.

From (33), Lemma 2.3 and the theorem on logarithmic derivatives, we have 


$$
\begin{aligned}
T\left(r, c_{j}\right) \leq & O\left(\sum_{i=0}^{k-1} T\left(r, Q_{1}^{(i)}\right)+\sum_{j=0}^{k-1} m\left(r, a_{j}\right)+\sum_{j=0}^{k-1} m\left(r, a_{j}^{\prime}\right)\right. \\
& +\sum_{s=0}^{k-1} m\left(r, P_{1}^{(s)}\right)+\sum_{t=1}^{k-2} m\left(r, U^{(t)} / U\right)+m(r, U) \\
& +\bar{N}(r, 1 / \pi)+O(\log r)) \\
\leq & O\left(r^{\xi_{3}}\right), \quad r \rightarrow \infty, j=0,1, \ldots, k-1,
\end{aligned}
$$

and

$$
\begin{aligned}
T\left(r, e^{P_{2}}\left[Q_{2}\left(Q_{1}^{\prime}+Q_{1} P_{1}^{\prime}\right)\right.\right. & \left.-Q_{1}\left(Q_{2}^{\prime}+Q_{2} P_{2}^{\prime}\right)\right] \\
& \left.+e^{P_{3}}\left[Q_{3}\left(Q_{1}^{\prime}+Q_{1} P_{1}^{\prime}\right)-Q_{1}\left(Q_{3}^{\prime}+Q_{3} P_{3}^{\prime}\right)\right]\right) \\
\leq & O\left(r^{\xi_{3}}\right)+T\left(r, e^{P_{2}}\right)+T\left(r, e^{P_{3}}\right) \\
= & (1+\lambda) T\left(r, e^{P_{2}}\right)+O\left(r^{\xi_{3}}\right) \\
\leq & (1+\lambda) \rho T\left(r, e^{P_{1}}\right)+O\left(r^{\xi_{3}}\right), \quad r \rightarrow \infty .
\end{aligned}
$$

From (27), (30), (34) and (35), we get

$$
\begin{aligned}
\frac{1-\varepsilon}{k} T\left(r, e^{P_{1}}\right)+ & O\left(r^{\xi_{3}}\right) \leq T\left(r, h^{\prime}\right) \\
& \leq(1+\lambda) \rho T\left(r, e^{P_{1}}\right)+O\left(r^{\xi_{3}}\right), \quad r \rightarrow \infty, r \notin E .
\end{aligned}
$$

Thus (36) implies

$$
\left(\frac{1-\varepsilon}{k}-(1+\lambda) \rho-o(1)\right) T\left(e, e^{P_{1}}\right) \leq 0, \quad r \rightarrow \infty, r \notin E .
$$

Since $0<\rho=\zeta_{2} / \zeta_{1}<1 / 2 k, 0<\lambda<1$, we get a contradiction. Hence $c_{k-1}=\cdots=c_{1}=c_{0}+e^{P_{2}}\left[Q_{2}\left(Q_{1}^{\prime}+Q_{1} P_{1}^{\prime}\right)-Q_{1}\left(Q_{2}^{\prime}+Q_{2} P_{2}^{\prime}\right)\right]+e^{P_{3}}\left[Q_{3}\left(Q_{1}^{\prime}+\right.\right.$ $\left.\left.Q_{1} P_{1}^{\prime}\right)-Q_{1}\left(Q_{3}^{\prime}+Q_{3} P_{3}^{\prime}\right)\right] \equiv 0$. From $(26)$, we have

$$
\begin{aligned}
-c_{0}(z)= & e^{P_{2}}\left[Q_{2}\left(Q_{1}^{\prime}+Q_{1} P_{1}^{\prime}\right)-Q_{1}\left(Q_{2}^{\prime}+Q_{2} P_{2}^{\prime}\right)\right] \\
& +e^{P_{3}}\left[Q_{3}\left(Q_{1}^{\prime}+Q_{1} P_{1}^{\prime}\right)-Q_{1}\left(Q_{3}^{\prime}+Q_{3} P_{3}^{\prime}\right)\right] .
\end{aligned}
$$

We assume that the right hand side above is not identically zero; otherwise, we have

$$
e^{P_{2}-P_{3}}=-\frac{Q_{3}\left(Q_{1}^{\prime}+Q_{1} P_{1}^{\prime}\right)-Q_{1}\left(Q_{3}^{\prime}+Q_{3} P_{3}^{\prime}\right)}{Q_{2}\left(Q_{1}^{\prime}+Q_{1} P_{1}^{\prime}\right)-Q_{1}\left(Q_{2}^{\prime}+Q_{2} P_{2}^{\prime}\right)},
$$

and since $\zeta_{3}=\lambda \zeta_{2}, 0<\lambda<1$, a simple order consideration leads to a contradiction. From (38), by (34) and Lemma 2.5, we obtain

$$
(1-\varepsilon) T\left(r, e^{P_{2}}\right)+O\left(r^{\xi_{3}}\right) \leq O\left(r^{\xi_{3}}\right), \quad r \rightarrow \infty .
$$

From (39), we have $\sigma\left(e^{P_{2}}\right)<\xi_{3}<n$, a contradiction. Hence $\lambda(f) \geq n$. 
Acknowledgments. We thank the referee(s) for reading the manuscript very carefully and making a number of valuable and kind comments which improved the presentation.

The research was supported by the NNSF of China (No. 10871108), the NSF of Jiangxi of China (2008GQS0075) and the Soft Scientific and Technological Research Projects of Jiangxi Province ([2008]147).

\section{References}

[1] R. P. Boas, Entire Functions, Academic Press, New York, 1954.

[2] Z. X. Chen, The growth of solutions of the differential equation $f^{\prime \prime}+e^{z} f^{\prime}+Q(z) f=0$, Sci. China Ser. A 31 (2001), 775-784 (in Chinese).

[3] - On the hyper order of solutions of some second order linear differential equations, Acta Math. Sinica B 18 (2002), 79-88.

[4] G. G. Gundersen, Estimates for the logarithmic derivative of a meromorphic function, plus similar estimates, J. London Math. Soc. 37 (1988), 88-104.

[5] W. Hayman, Meromorphic Functions, Clarendon Press, Oxford, 1964.

[6] K. Ishizaki, An oscillation result for a certain linear differential equation of second order, Hokkaido Math. J. 26 (1997), 421-434.

[7] K. Ishizaki and K. Tohge, On the complex oscillation of some linear differential equations, J. Math. Anal. Appl. 206 (1997), 503-517.

[8] I. Laine, Nevanlinna Theory and Complex Differential Equations, de Gruyter, Berlin, 1993.

[9] A. I. Markushevich, Theory of Functions of a Complex Variable, Vol. 2, PrenticeHall, Englewood Cliffs, NJ, 1965.

[10] J. Tu and Z. X. Chen, Zeros of solutions of certain second order linear differential equation, J. Math. Anal. Appl. 332 (2007), 279-291.

[11] J. Wang and Z. X. Chen, Zeros of solutions of higher order linear differential equations, J. Systems Sci. Math. Sci. 21 (2001), 314-324 (in Chinese).

Hong-Yan $\mathrm{Xu}$ (corresponding author)

Department of Informatics and Engineering

Jingdezhen Ceramic Institute (XiangHu XiaoQu)

Jingdezhen, Jiangxi 333403, China

E-mail: xhyhhh@126.com
Cai-Feng Yi

Institute of Mathematics and Informatics

Jiangxi Normal University Nanchang, Jiangxi 330027, China E-mail: yicaifeng55@163.com

Received 27.2.2009

and in final form 14.4.2009 